\title{
Asymptotic behavior of third-order functional differential equations with a negative middle term
}

\author{
Jozef Džurina and Irena Jadlovská*
}

\author{
"Correspondence: \\ irena.jadlovska@tuke.sk \\ Department of Mathematics and \\ Theoretical Informatics, Faculty of \\ Electrical Engineering and \\ Informatics, Technical University of \\ Košice, Letná 9, Košice, 042 00, \\ Slovakia
}

\begin{abstract}
This paper is concerned with asymptotic and oscillatory properties of the nonlinear third-order differential equation with a negative middle term. Both delay and advanced cases of argument deviation are considered. Sufficient conditions for all solutions of a given differential equation to have property $\mathrm{B}$ or to be oscillatory are established. A couple of illustrative examples is also included.
\end{abstract}

MSC: $34 \mathrm{~K} 11$

Keywords: third-order differential equation; delay; advance; oscillation; comparison; integral criteria

\section{Introduction}

The purpose of this work is to investigate the asymptotic behavior of the third-order functional differential equation with the middle-term

$$
\left(r_{2}(t)\left(r_{1}(t)\left(y^{\prime}(t)\right)^{\gamma}\right)^{\prime}\right)^{\prime}-p(t)\left(y^{\prime}(t)\right)^{\gamma}-q(t) f(y(g(t)))=0, \quad t \geq t_{0}
$$

where $t_{0}$ is fixed and $\gamma$ is a quotient of odd positive integers. Throughout this paper, we assume that

(i) $r_{1}, r_{2}, q \in C(\mathcal{I},(0, \infty))$, where $\mathcal{I}=\left[t_{0}, \infty\right)$;

(ii) $p \in C(\mathcal{I},[0, \infty))$;

(iii) $g \in C^{1}(\mathcal{I}, \mathbb{R}), g^{\prime}(t) \geq 0, \lim _{t \rightarrow \infty} g(t)=\infty$;

(iv) $f \in C^{1}(\mathbb{R}, \mathbb{R}), x f(x)>0, f^{\prime}(x) \geq 0$ for $x \neq 0, f(x y) \geq f(x) f(y)$ for $x y>0$.

By a solution of equation (1.1) we mean a function $y \in C^{1}\left(\left[T_{y}, \infty\right)\right), T_{y} \in \mathcal{I}$, which has the property $r_{1} y^{\prime}, r_{2}\left(r_{1}\left(y^{\prime}\right)^{\gamma}\right)^{\prime} \in C^{1}\left(\left[T_{y}, \infty\right), \mathbb{R}\right)$ and satisfies $(1.1)$ on $\left[T_{y}, \infty\right)$. Our attention is restricted to those solutions $y$ of (1.1) which exist on $\mathcal{I}$ and satisfy the condition

$$
\sup \left\{|y(t)|: t_{1} \leq t<\infty\right\}>0 \text { for any } t_{1} \geq t_{0}
$$

We make the standing hypothesis that (1.1) admits such a solution. A solution of (1.1) is called oscillatory if it has arbitrarily large zeros on $\left[T_{y}, \infty\right)$ and otherwise it is called nonoscillatory. Equation (1.1) is said to be oscillatory if all its solutions are oscillatory.

(c) The Author(s) 2017. This article is distributed under the terms of the Creative Commons Attribution 4.0 International License (http://creativecommons.org/licenses/by/4.0/), which permits unrestricted use, distribution, and reproduction in any medium, provided you give appropriate credit to the original author(s) and the source, provide a link to the Creative Commons license, and indicate if changes were made. 
Analysis of the asymptotic and oscillatory behavior of solutions to different classes of differential and functional differential equations has experienced long-term interest of many researchers, see, for example, [1-23] and the references cited therein. A huge amount of significant oscillation results has been collected in several excellent monographs, see, e.g., $[1,2,16,21]$. This interest is caused by the fact that differential equations, especially those with deviating argument, are deemed to be adequate in modeling of countless processes in all areas of science. In particular, it is worthwhile to mention the use of third-order differential equations in the study of an entry-flow phenomenon in a problem of hydrodynamics, or of the propagation of electrical pulses in the nerve of a squid approximated by the famous Nagumo's equation [21].

In the recent works [8,9], the authors used a generalized Riccati transformation and an integral averaging technique in order to establish some sufficient conditions for oscillation of all solutions of a trinomial third-order differential equation

$$
\left(r_{2}(t)\left(r_{1}(t)\left(y^{\prime}(t)\right)^{\gamma}\right)^{\prime}\right)^{\prime}+p(t)\left(y^{\prime}(t)\right)^{\gamma}+q(t) f(y(g(t)))=0
$$

where $p(t)$ and $q(t)$ are positive functions and the auxiliary equation

$$
\left(r_{2}(t) z^{\prime}(t)\right)^{\prime}+\frac{p(t)}{r_{1}(t)} z(t)=0
$$

is nonoscillatory. They have shown (see [8], Lemma 2.2) that any nonoscillatory solution of (1.2) satisfies

$$
y(t) y^{\prime}(t)<0 \quad \text { or } \quad y(t) y^{\prime}(t)>0 .
$$

Another approach for studying the asymptotic properties of (1.2) has been employed in papers $[6,11]$ when $p(t)$ is negative and $q(t)$ is positive. The authors presented several comparison theorems in which the desired properties of solutions are deduced from those of corresponding first-order functional or second-order ordinary differential equations. Their results, however, strongly rely on the knowledge of the auxiliary solution $z(t)$.

In this work, we would like to study equation (1.1) under assumptions (i)-(v). The organization of the paper is as follows. Using different arguments as those in [8] and by imposing one restrictive condition on coefficients of the corresponding auxiliary equation, we show that any nonoscillatory solution $y(t)$ of (1.1) satisfies

$$
y(t) y^{\prime}(t)>0 .
$$

In the next, we consider separately delay and advanced cases of the argument deviation to establish new sufficient conditions for all solutions of (1.1) to have property B (see Definition 1). Moreover, in the advanced case, we will attain oscillation of all solutions of (1.1).

\section{Some basic definitions and auxiliary lemmas}

Following [8], we define

$$
L_{0} y(t)=y(t), \quad L_{1} y(t)=r_{1}(t)\left(y^{\prime}(t)\right)^{\gamma},
$$




$$
L_{2} y(t)=r_{2}(t)\left(L_{1} y(t)\right)^{\prime}, \quad L_{3} y(t)=\left(L_{2} y(t)\right)^{\prime},
$$

for $t \in \mathcal{I}$. With this notation, (1.1) can be rewritten as

$$
L_{3} y(t)-\frac{p(t)}{r_{1}(t)} L_{1} y(t)-q(t) f(y(g(t)))=0
$$

For the sake of clarity, we list the functions used in this work:

$$
\begin{aligned}
& R_{1}\left(t, t_{1}\right)=\int_{t_{1}}^{t} \frac{1}{r_{1}^{1 / \gamma}(s)} \mathrm{d} s, \quad R_{2}\left(t, t_{1}\right)=\int_{t_{1}}^{t} \frac{1}{r_{2}(s)} \mathrm{d} s, \quad R_{12}\left(t, t_{1}\right)=\int_{t_{1}}^{t} \frac{R_{2}^{1 / \gamma}\left(s, t_{1}\right)}{r_{1}^{1 / \gamma}(s)} \mathrm{d} s, \\
& P(t)=\exp \left(-\int_{t_{1}}^{t} \frac{1}{r_{2}(s)} \int_{s}^{\infty} \frac{p(u)}{r_{1}(u)} \mathrm{d} u \mathrm{~d} s\right), \quad \tilde{P}(t)=\int_{t}^{\infty} \frac{1}{r_{2}(s)} \int_{s}^{\infty} q(u) \mathrm{d} u \mathrm{~d} s, \\
& Q(t)=\frac{p(t)}{r_{1}(t)} \tilde{P}(t)+q(t), \quad \tilde{Q}(t)=\frac{1}{r_{2}(t)} \int_{t}^{\infty} Q(s) \mathrm{d} s, \\
& \tilde{q}(s, t)=\int_{t}^{s} \frac{1}{r_{2}(u)} \int_{t}^{u} q(x) f\left(R_{12}\left(g(x), t_{1}\right)\right) \mathrm{d} x \mathrm{~d} u, \\
& R(s, t)=\frac{p(s)}{r_{1}(s)} \tilde{q}(s, t)+q(s) f\left(R_{12}\left(g(s), t_{1}\right)\right),
\end{aligned}
$$

for $s \geq t \geq t_{1}, t_{1} \in \mathcal{I}$.

Throughout and without further mentioning, it will be assumed that

$$
R_{i}\left(t, t_{0}\right) \rightarrow \infty \quad \text { as } t \rightarrow \infty, \text { for } i=1,2 \text {, }
$$

which means that the operator $L_{3} y(t)$ is in the so-called canonical form (see Trench [24]).

To give a sense of the definitions of $P(t)$ and $\tilde{P}(t)$, we also suppose that

$$
\int_{t_{0}}^{\infty} \frac{p(s)}{r_{1}(s)} \mathrm{d} s<\infty \text { and } \int_{t_{0}}^{\infty} q(s) \mathrm{d} s<\infty
$$

Remark 1 All the functional inequalities considered in this paper are assumed to hold eventually, that is, they are satisfied for all $t$ large enough.

Remark 2 In the sequel and without loss of generality, we can restrict our attention only to positive solutions of (1.1).

Properties of solutions to equation (1.1) are closely related to those of solutions to an auxiliary second-order linear ordinary differential equation

$$
\left(r_{2}(t) v^{\prime}(t)\right)^{\prime}=\frac{p(t)}{r_{1}(t)} v(t)
$$

as the following lemma says.

Lemma 1 Let $v(t)$ be a positive solution of (2.1) on $\mathcal{I}$. Then (1.1) can be written in the form

$$
\left(r_{2}(t) v^{2}(t)\left(\frac{r_{1}(t)}{v(t)}\left(y^{\prime}(t)\right)^{\gamma}\right)^{\prime}\right)^{\prime}=q(t) v(t) f(y(g(t))), \quad t \in \mathcal{I} .
$$


Proof It follows from a straightforward calculation that

$$
\left(r_{2}(t)\left(r_{1}(t)\left(y^{\prime}(t)\right)^{\gamma}\right)^{\prime}\right)^{\prime}-p(t)\left(y^{\prime}(t)\right)^{\gamma}=\frac{1}{v(t)}\left(r_{2}(t) v^{2}(t)\left(\frac{r_{1}(t)}{v(t)}\left(y^{\prime}(t)\right)^{\gamma}\right)^{\prime}\right)^{\prime}
$$

The proof of the above equivalence is similar to that stated in [5], and so it is omitted.

We recall that (2.1) always has a couple of nonoscillatory solutions such that, for all $t \in \mathcal{I}$, either

$$
v(t) v^{\prime}(t)>0
$$

or

$$
v(t) v^{\prime}(t)<0
$$

According to a classical work of Hartman [15], a nonoscillatory solution $v(t)$ of (2.1) satisfying (2.4) is termed a principal solution at infinity, and such a solution is determined uniquely up to a constant factor. In order to reveal the structure of possible nonoscillatory solutions of (1.1), the following property of a principal solution of (2.1) plays a crucial role.

\section{Lemma 2 If}

$$
\int_{t_{1}}^{\infty}\left(\frac{P(s)}{r_{1}(s)}\right)^{1 / \gamma} \mathrm{d} s=\infty
$$

then (2.1) has a positive solution $v(t)$ satisfying

$$
\int_{t_{1}}^{\infty} \frac{1}{r_{2}(s) v^{2}(s)} \mathrm{d} s=\int_{t_{1}}^{\infty}\left(\frac{v(s)}{r_{1}(s)}\right)^{1 / \gamma} \mathrm{d} s=\infty
$$

Proof Let $v(t)$ be a principal solution of (2.1) which is positive on $\left[t_{1}, \infty\right)$. It is clear from the fact that $v^{\prime}(t)<0$ and the assumption (v) that the first integral in (2.6) is divergent. On the other hand, since

$$
\left(r_{2}(t) v^{\prime}(t)\right)^{\prime}=\frac{p(t)}{r_{1}(t)} v(t) \geq 0
$$

then $r_{2}(t) v^{\prime}(t)$ is increasing and there exists a constant $\ell \leq 0$ such that

$$
\lim _{t \rightarrow \infty} r_{2}(t) v^{\prime}(t)=\ell
$$

We claim that $\ell=0$. If not, then

$$
v(t) \leq v\left(t_{1}\right)+\ell \int_{t_{1}}^{t} r_{2}^{-1}(s) \mathrm{d} s \rightarrow-\infty \quad \text { as } t \rightarrow \infty,
$$

a contradiction. Thus $\ell=0$. By integrating (2.1) from $t$ to $\infty$, we see that

$$
-r_{2}(t) v^{\prime}(t)=\int_{t}^{\infty} \frac{p(s)}{r_{1}(s)} v(s) \mathrm{d} s \leq v(t) \int_{t}^{\infty} \frac{p(s)}{r_{1}(s)} \mathrm{d} s
$$


Integrating (2.7) from $t_{1}$ to $t$, we get

$$
v(t) \geq v\left(t_{1}\right) \exp \left(-\int_{t_{1}}^{t} \frac{1}{r_{2}(s)} \int_{s}^{\infty} \frac{p(u)}{r_{1}(u)} \mathrm{d} u \mathrm{~d} s\right),
$$

that yields

$$
\left(\frac{v(t)}{r_{1}(t)}\right)^{1 / \gamma} \geq v^{1 / \gamma}\left(t_{1}\right)\left(\frac{P(t)}{r_{1}(t)}\right)^{1 / \gamma}
$$

It is easy to see that integration of (2.8) from $t_{1}$ to $\infty$ together with (2.5) implies that the second integral in (2.6) is divergent. The proof is complete.

In the lemma below we recall the adaptation of the generalized Kiguradze lemma [17] to the canonical operator $L_{3} y(t)$.

Lemma 3 Let $y(t)$ be a real-valued function on $\mathcal{I}$ which has the property $L_{n} y(t) \in \mathcal{C}^{1}(\mathcal{I})$, $n=0,1,2$. If

$$
y(t) L_{3} y(t)>0 \quad \text { on } \mathcal{I}
$$

then there exists $t_{1} \in \mathcal{I}$ and $\ell=\{1,3\}$ such that

$$
\begin{aligned}
& y(t) L_{j} y(t)>0, \quad 0 \leq j \leq \ell, \\
& (-1)^{j-\ell} y(t) L_{j} y(t)>0, \quad \ell+1 \leq j \leq 3, \text { on }\left[t_{1}, \infty\right) .
\end{aligned}
$$

Now we are prepared to state the sign structure of possible nonoscillatory solutions to equation (1.1). We introduce the following classes of nonoscillatory (let us say positive) solutions:

$$
\begin{aligned}
& y(t) \in \mathcal{N}_{1} \Longleftrightarrow y(t)>0, \quad L_{1} y(t)>0, \quad L_{2} y(t)<0, \quad L_{3} y(t)>0, \\
& y(t) \in \mathcal{N}_{3} \Longleftrightarrow y(t)>0, \quad L_{1} y(t)>0, \quad L_{2} y(t)>0, \quad L_{3} y(t)>0,
\end{aligned}
$$

for $t \geq t_{1}$.

Lemma 4 Assume that (2.5) holds. If $y(t)$ is a positive solution of (1.1) on $\mathcal{I}$, then there exists $t_{1} \in \mathcal{I}$ such that either $y(t) \in \mathcal{N}_{1}$ or $y(t) \in \mathcal{N}_{3}$ on $\left[t_{1}, \infty\right)$.

Proof Assume that $y(t)$ is a positive solution of (1.1) on $\mathcal{I}$. As a consequence of Lemma 1, we may rewrite (1.1) in an equivalent binomial form (2.2). In view of Lemma 2, there exists a positive solution $v(t)$ of (2.1) which satisfies (2.6); and therefore, we see that the operator

$$
\left(r_{2}(t) v^{2}(t)\left(\frac{r_{1}(t)}{v(t)}\left(y^{\prime}(t)\right)^{\gamma}\right)^{\prime}\right)^{\prime}>0
$$

is in a canonical form. Then, by Lemma 3, $y(t)$ satisfies either

$$
y(t)>0, \quad \frac{r_{1}(t)}{v(t)}\left(y^{\prime}(t)\right)^{\gamma}>0, \quad r_{2}(t) v^{2}(t)\left(\frac{r_{1}(t)}{v(t)}\left(y^{\prime}(t)\right)^{\gamma}\right)^{\prime}<0,
$$


or

$$
y(t)>0, \quad \frac{r_{1}(t)}{v(t)}\left(y^{\prime}(t)\right)^{\gamma}>0, \quad r_{2}(t) v^{2}(t)\left(\frac{r_{1}(t)}{v(t)}\left(y^{\prime}(t)\right)^{\gamma}\right)^{\prime}>0,
$$

for $t \geq t_{1}$. Note that in both cases we have $y^{\prime}(t)>0$ and, by virtue of (1.1), we can see that $L_{3} y(t)>0$. The rest sign properties of quasi-derivatives $L_{i} y(t), i=1,2$, immediately follow from Lemma 3.

Consequently, if we assume (2.5), the set $\mathcal{N}$ of all positive solutions of (1.1) has the following decomposition:

$$
\mathcal{N}=\mathcal{N}_{1} \cup \mathcal{N}_{3} .
$$

According to the well-known results of Kiguradze and Chanturia [16], the oscillation criteria are often accomplished by introducing the concepts of having property $A$ and/or $B$. Such properties have been widely studied by many authors, see, e.g., $[4,10,16,19]$ and the references cited therein.

Definition 1 Equation (1.1) is said to have Property B if $\mathcal{N}=\mathcal{N}_{3}$.

In what follows, we state and prove some useful estimates which will play an important role in the proofs of our main results.

Lemma 5 Let $y(t) \in \mathcal{N}_{1}$ be a positive solution of $(1.1)$ on $\left[t_{1}, \infty\right)$. Then

$$
\begin{aligned}
& \frac{y(t)}{R_{1}\left(t, t_{1}\right)} \quad \text { is nonincreasing, } \\
& L_{1} y(t) \geq \tilde{P}(t) f(y(g(t)))
\end{aligned}
$$

for $t \geq t_{1}$.

Proof Assume that $y(t) \in \mathcal{N}_{1}$ is a positive solution of (1.1) for $t \geq t_{1}$. It follows from the monotonicity of $L_{1} y(t)$ that

$$
y(t) \geq y(t)-y\left(t_{1}\right)=\int_{t_{1}}^{t} r_{1}^{-1 / \gamma}(s) L_{1}^{1 / \gamma} y(s) \mathrm{d} s \geq R_{1}\left(t, t_{1}\right) L_{1}^{1 / \gamma} y(t) .
$$

Therefore,

$$
\left(\frac{y(t)}{R_{1}\left(t, t_{1}\right)}\right)^{\prime}=\frac{L_{1}^{1 / \gamma} y(t) R_{1}\left(t, t_{1}\right)-y(t)}{r^{1 / \gamma}(t) R_{1}^{2}\left(t, t_{1}\right)} \leq 0,
$$

and so $y(t) / R_{1}\left(t, t_{1}\right)$ is nonincreasing.

On the other hand, integration of (1.1) from $t$ to $\infty$ yields

$$
\begin{aligned}
-L_{2} y(t) & \geq \int_{t}^{\infty} \frac{p(s)}{r_{1}(s)} L_{1} y(s) \mathrm{d} s+\int_{t}^{\infty} q(s) f(y(g(s))) \mathrm{d} s \\
& \geq f(y(g(t))) \int_{t}^{\infty} q(s) \mathrm{d} s .
\end{aligned}
$$


By repeated integration, we obtain

$$
L_{1} y(t) \geq \int_{t}^{\infty} \frac{f(y(g(s)))}{r_{2}(s)} \int_{s}^{\infty} q(u) \mathrm{d} u \mathrm{~d} s \geq \tilde{P}(t) f(y(g(t))) .
$$

The proof is complete.

In the lemma below we shall point out that estimate (2.10) can be improved further.

Define

$$
\begin{aligned}
& P_{0}(t)=\tilde{P}(t), \\
& P_{n}(t)=\int_{t}^{\infty} \frac{1}{r_{2}(s)} \int_{s}^{\infty} \frac{p(u)}{r_{1}(u)} P_{n-1}(u) \mathrm{d} u \mathrm{~d} s+P_{0}(t), \quad t \geq t_{0}, n \in \mathbb{N} .
\end{aligned}
$$

Lemma 6 Let $y(t) \in \mathcal{N}_{1}$ be a solution of $(1.1)$ on $\left[t_{1}, \infty\right), t_{1} \in \mathcal{I}$. Then

$$
L_{1} y(t) \geq P_{n}(t) f(y(g(t))), \quad t \geq t_{1}, n \in \mathbb{N}
$$

Proof Proceeding as in the proof of Lemma 5, we obtain (2.10). Setting (2.10) into (1.1) and integrating twice from $t$ to $\infty$, we see that

$$
L_{1} y(t) \geq\left(\int_{t}^{\infty} \frac{1}{r_{2}(s)} \int_{s}^{\infty} \frac{p(u)}{r_{1}(u)} P_{0}(u) \mathrm{d} u \mathrm{~d} s+P_{0}(t)\right) f(y(g(t)))=P_{1}(t) f(y(g(t))) .
$$

By induction, we can show that (2.12) holds for any $n \in \mathbb{N}$.

Lemma 7 Let $y(t) \in \mathcal{N}_{3}$ be a positive solution of $(1.1)$ on $\left[t_{1}, \infty\right)$. If

$$
\int_{t_{1}}^{\infty} \frac{p(s)}{r_{1}(s)} R_{2}\left(s, t_{1}\right)+q(s) f\left(R_{12}\left(g\left(s, t_{1}\right)\right)\right) \mathrm{d} s=\infty
$$

then there exists $t_{2}>t_{1}$ such that

$$
\frac{y(t)}{R_{12}\left(t, t_{1}\right)} \quad \text { is nondecreasing on }\left[t_{2}, \infty\right) \text {. }
$$

Proof Assume that $y(t) \in \mathcal{N}_{3}$ is a positive solution of (1.1) for $t \geq t_{1}$. Since $L_{2} y(t)$ is increasing, $L_{2} y(t) \geq L_{2} y\left(t_{1}\right)=: \ell$. Obviously,

$$
L_{1} y(t) \geq \ell R_{2}\left(t, t_{1}\right) \quad \text { and } \quad y(t) \geq \ell^{1 / \gamma} R_{12}\left(t, t_{1}\right) \quad \text { for } t \geq t_{1} .
$$

We claim that (2.13) implies $\lim _{t \rightarrow \infty} L_{2} y(t)=\infty$. Setting the above estimates into (1.1), we obtain

$$
L_{3} y(t) \geq \ell \frac{p(t)}{r_{1}(t)} R_{2}\left(t, t_{1}\right)+f\left(\ell^{1 / \gamma}\right) q(t) f\left(R_{12}\left(g(t), t_{1}\right)\right) .
$$


By integrating (2.15) from $t_{1}$ to $\infty$, we see that the claim holds. Therefore, for any $t \geq t_{2}>t_{1}$,

$$
\begin{aligned}
L_{1} y(t) & =L_{1} y\left(t_{2}\right)+\int_{t_{2}}^{t} r_{2}^{-1}(s) L_{2} y(s) \mathrm{d} s \leq L_{1} y\left(t_{2}\right)+L_{2} y(t) R_{2}\left(t, t_{2}\right) \\
& =L_{1} y\left(t_{2}\right)-R_{2}\left(t_{2}, t_{1}\right) L_{2} y(t)+R_{2}\left(t, t_{1}\right) L_{2} y(t) \\
& \leq R_{2}\left(t, t_{1}\right) L_{2} y(t),
\end{aligned}
$$

which leads to

$$
\left(\frac{L_{1} y(t)}{R_{2}\left(t, t_{1}\right)}\right)^{\prime}=\frac{L_{2} y(t) R_{2}\left(t, t_{1}\right)-L_{1} y(t)}{r_{2}(t) R_{2}^{2}\left(t, t_{1}\right)} \geq 0
$$

and consequently, $L_{1} y(t) / R_{2}\left(t, t_{1}\right)$ is nondecreasing on $\left[t_{2}, \infty\right)$.

In the same way, for any $t \geq t_{3}>t_{2}$,

$$
\begin{aligned}
y(t) & =y\left(t_{3}\right)+\int_{t_{3}}^{t}\left(\frac{R_{2}\left(s, t_{1}\right)}{r_{1}(s)} \frac{L_{1} y(s)}{R_{2}\left(s, t_{1}\right)}\right)^{1 / \gamma} \mathrm{d} s \leq y\left(t_{3}\right)+R_{12}\left(t, t_{3}\right)\left(\frac{L_{1} y(t)}{R_{2}\left(t, t_{1}\right)}\right)^{1 / \gamma} \\
& \leq y\left(t_{3}\right)-R_{12}\left(t_{3}, t_{1}\right)\left(\frac{L_{1} y(t)}{R_{2}\left(t, t_{1}\right)}\right)^{1 / \gamma}+R_{12}\left(t, t_{1}\right)\left(\frac{L_{1} y(t)}{R_{2}\left(t, t_{1}\right)}\right)^{1 / \gamma} .
\end{aligned}
$$

It follows from l'Hospital's rule that $\lim _{t \rightarrow \infty} L_{1} y(t) / R_{2}\left(t, t_{1}\right)=\lim _{t \rightarrow \infty} L_{2} y(t)=\infty$, and so we have

$$
y(t) \leq R_{12}\left(t, t_{1}\right)\left(\frac{L_{1} y(t)}{R_{2}\left(t, t_{1}\right)}\right)^{1 / \gamma}, \quad t \geq t_{3} .
$$

Then

$$
\left(\frac{y(t)}{R_{12}\left(t, t_{1}\right)}\right)^{\prime}=\frac{\left(L_{1} y(t)\right)^{1 / \gamma} R_{12}\left(t, t_{1}\right)-\left(R_{2}\left(t, t_{1}\right)\right)^{1 / \gamma} y(t)}{r_{1}^{1 / \gamma}(t) R_{12}^{2}\left(t, t_{1}\right)} \geq 0 .
$$

Thus $y(t) / R_{12}\left(t, t_{1}\right)$ is nondecreasing on $\left[t_{3}, \infty\right)$. The proof is complete now.

Remark 3 It is easy to see that if (1.1) has Property B, then any positive solution of (1.1) satisfies

$$
\lim _{t \rightarrow \infty} \frac{y(t)}{R_{12}\left(t, t_{1}\right)}=\infty
$$

which gives us information about the rate of convergence of possible positive solutions.

We conclude the introductory part by recalling a useful relationship between the existence of positive solutions of the first-order functional differential inequalities

$$
\begin{aligned}
& y^{\prime}(t)+q(t) f(y(g(t))) \leq 0, \\
& y^{\prime}(t)-q(t) f(y(g(t))) \geq 0,
\end{aligned}
$$


and the corresponding first-order functional differential equations

$$
\begin{aligned}
& y^{\prime}(t)+q(t) f(y(g(t)))=0, \\
& y^{\prime}(t)-q(t) f(y(g(t)))=0,
\end{aligned}
$$

where $q, g$ and $f$ satisfy conditions (i), (iii) and (iv), respectively. The following lemma can be found in [1] or, separately for delayed and advanced cases, in [22] and [3], respectively.

Lemma 8 Let $g(t)<t(g(t)>t)$. If inequality (2.16) (inequality (2.17)) has an eventually positive solution, then so does equation (2.18) (equation (2.19)).

\section{Main results}

\subsection{Criteria for Property B}

Now we are prepared to give sufficient conditions under which (1.1) enjoys Property B. We distinguish between delayed and advanced types of the argument deviation.

Theorem 1 Let (2.5) hold and $g(t)<t$ for $t \geq t_{1}$. If the first-order delay differential equation

$$
z^{\prime}(t)+\tilde{Q}(t) f\left(R_{1}\left(g(t), t_{1}\right)\right) f\left(z^{1 / \gamma}(g(t))\right)=0
$$

is oscillatory, then (1.1) has Property B.

Proof Let $y(t)$ be a positive solution of (1.1) on $\mathcal{I}$. It follows from Lemma 4 that there exists $t_{1} \in \mathcal{I}$ such that either $y(t) \in \mathcal{N}_{1}$ or $y(t) \in \mathcal{N}_{3}$ on $\left[t_{1}, \infty\right)$. If $y(t) \in \mathcal{N}_{1}$, then by virtue of (1.1) and (2.10), we have

$$
L_{3} y(t) \geq\left(\frac{p(t)}{r_{1}(t)} \tilde{P}(t)+q(t)\right) f(y(g(t)))=Q(t) f(y(g(t))) .
$$

Integrating (3.2) from $t$ to $\infty$, we find

$$
-L_{2} y(t) \geq \int_{t}^{\infty} Q(s) f(y(g(s))) \mathrm{d} s \geq\left(\int_{t}^{\infty} Q(s) \mathrm{d} s\right) f(y(g(t)))
$$

Using (2.11) in the latter inequality yields

$$
-\left(L_{1} y(t)\right)^{\prime} \geq \frac{1}{r_{2}(t)}\left(\int_{t}^{\infty} Q(s) \mathrm{d} s\right) f\left(R_{1}\left(g(t), t_{1}\right)\right) f\left(L_{1}^{1 / \gamma} y(g(t))\right) .
$$

Letting $z(t)=L_{1} y(t)$, we see that the differential inequality

$$
z^{\prime}(t)+\tilde{Q}(t) f\left(R_{1}\left(g(t), t_{1}\right)\right) f\left(z^{1 / \gamma}(g(t))\right) \leq 0
$$

has a positive solution. By Lemma 8 , we see that (3.1) also has a positive solution, which contradicts the hypothesis. Therefore $y(t) \in \mathcal{N}_{3}$, which means that (1.1) has Property B. The proof is complete. 
Theorem 2 Let (2.5) hold and $g(t)>t$ for $t \geq t_{1}$. If the first-order advanced differential equation

$$
y^{\prime}(t)-\frac{1}{r_{1}^{1 / \gamma}(t)}\left(\int_{t}^{\infty} \tilde{Q}(s) \mathrm{d} s\right)^{1 / \gamma} f^{1 / \gamma}(y(g(t)))=0
$$

is oscillatory, then (1.1) has Property B.

Proof Let $y(t)$ be a positive solution of (1.1) on $\mathcal{I}$. It follows from Lemma 4 that there exists $t_{1} \in \mathcal{I}$ such that either $y(t) \in \mathcal{N}_{1}$ or $y(t) \in \mathcal{N}_{3}$ on $\left[t_{1}, \infty\right)$. If $y(t) \in \mathcal{N}_{1}$, then, as in the proof of Theorem 1, we obtain (3.3) so that by integration from over $[t, \infty)$, we find that

$$
L_{1} y(t) \geq \int_{t}^{\infty} \frac{f(y(g(u)))}{r_{2}(u)} \int_{u}^{\infty} Q(s) \mathrm{d} s \mathrm{~d} u \geq\left(\int_{t}^{\infty} \tilde{Q}(s) \mathrm{d} s\right) f(y(g(t))) .
$$

Therefore, it is clear that $y(t)$ is a positive solution of the advanced differential inequality

$$
y^{\prime}(t)-\frac{1}{r_{1}^{1 / \gamma}(t)}\left(\int_{t}^{\infty} \tilde{Q}(s) \mathrm{d} s\right)^{1 / \gamma} f^{1 / \gamma}(y(g(t))) \geq 0
$$

By Lemma 8, we see that (3.4) also has a positive solution, a contradiction. Therefore $y(t) \in$ $\mathcal{N}_{3}$, which means that (1.1) has Property B. The proof is complete.

Employing some known criteria for oscillation of first-order functional differential equations (3.1) and (3.4), one can easily obtain oscillation criteria for (1.1). The following ones are due to Ladde et al. [20].

Corollary 1 Assume that $f(u)=u^{\gamma}$. Let (2.5) hold and $g(t)<t$ for $t \geq t_{1}$. If

$$
\liminf _{t \rightarrow \infty} \int_{g(t)}^{t} \tilde{Q}(u) R_{1}^{\gamma}\left(g(u), t_{1}\right) \mathrm{d} u>\frac{1}{e}
$$

then (1.1) has Property B.

Corollary 2 Assume that $f(u)=u^{\gamma}$. Let (2.5) hold and $g(t)>t$ for $t \geq t_{1}$. If

$$
\liminf _{t \rightarrow \infty} \int_{t}^{g(t)} \frac{1}{r_{1}^{1 / \gamma}(u)}\left(\int_{u}^{\infty} \tilde{Q}(s) \mathrm{d} s\right)^{1 / \gamma} \mathrm{d} u>\frac{1}{e},
$$

then (1.1) has Property B.

Now, we present other results for (1.1) to have Property B which are applicable even in the ordinary case $g(t)=t$.

Theorem 3 Let (2.5) hold and $g(t) \leq t$ for $t \geq t_{1}$. Assume that

$$
\int_{t_{1}}^{\infty} \frac{1}{r_{1}^{1 / \gamma}(v)}\left(\int_{v}^{\infty} \frac{1}{r_{2}(u)} \int_{u}^{\infty} Q(s) \mathrm{d} s \mathrm{~d} u\right)^{1 / \gamma} \mathrm{d} v=\infty
$$


and the function $f$ satisfies

$$
\lim _{x= \pm \infty} \frac{x}{f^{1 / \gamma}(x)}=k<\infty
$$

If

$$
\limsup _{t \rightarrow \infty}\left\{f^{1 / \gamma}\left(\frac{1}{R_{1}\left(t, t_{1}\right)}\right) \int_{t_{1}}^{t}\left(\frac{f\left(R_{1}\left(g(s), t_{1}\right)\right)}{r_{1}(s)} \int_{s}^{\infty} \tilde{Q}(u) \mathrm{d} u\right)^{1 / \gamma} \mathrm{d} s\right\}>k
$$

then (1.1) has Property B.

Proof Let $y(t)$ be a positive solution of (1.1) on $\mathcal{I}$. It follows from Lemma 4 that there exists $t_{1} \in \mathcal{I}$ such that either $y(t) \in \mathcal{N}_{1}$ or $y(t) \in \mathcal{N}_{3}$ on $\left[t_{1}, \infty\right)$. If $y(t) \in \mathcal{N}_{1}$, then, the same as in the proof of Theorem 2, we get (3.5). Then, by integrating (3.5) from $t_{1}$ to $t$, we easily find that

$$
y(t) \geq \int_{t_{1}}^{t}\left(\frac{f(y(g(s)))}{r_{1}(s)} \int_{s}^{\infty} \tilde{Q}(u) \mathrm{d} u\right)^{1 / \gamma} \mathrm{d} s .
$$

By virtue of the monotonicity property (2.9) and the fact that $g(t) \leq t$, we have

$$
\begin{aligned}
y(t) & \geq f^{1 / \gamma}\left(\frac{y(g(t))}{R_{1}\left(g(t), t_{1}\right)}\right) \int_{t_{1}}^{t}\left(\frac{f\left(R_{1}\left(g(s), t_{1}\right)\right)}{r_{1}(s)} \int_{s}^{\infty} \tilde{Q}(u) \mathrm{d} u\right)^{1 / \gamma} \mathrm{d} s \\
& \geq f^{1 / \gamma}\left(\frac{y(t)}{R_{1}\left(t, t_{1}\right)}\right) \int_{t_{1}}^{t}\left(\frac{f\left(R_{1}\left(g(s), t_{1}\right)\right)}{r_{1}(s)} \int_{s}^{\infty} \tilde{Q}(u) \mathrm{d} u\right)^{1 / \gamma} \mathrm{d} s .
\end{aligned}
$$

Using the assumption (iv) posed on the function $f$ and dividing both sides of the latter inequality by $f^{1 / \gamma} y(t)$, one can see that

$$
\frac{y(t)}{f^{1 / \gamma}(y(t))} \geq f^{1 / \gamma}\left(\frac{1}{R_{1}\left(t, t_{1}\right)}\right) \int_{t_{1}}^{t}\left(\frac{f\left(R_{1}\left(g(s), t_{1}\right)\right)}{r_{1}(s)} \int_{s}^{\infty} \tilde{Q}(u) \mathrm{d} u\right)^{1 / \gamma} \mathrm{d} s .
$$

It follows from (3.6) that $\lim _{t \rightarrow \infty} y(t)=\infty$. Taking the lim sup on both sides of (3.10), we are led to the contradiction with (3.8). Therefore $y(t) \in \mathcal{N}_{3}$, which means that (1.1) has Property B. The proof is complete.

Theorem 4 Let (2.5), (3.7) and (3.6) hold, and $g(t) \geq t$ for $t \geq t_{1}$. If

$$
\limsup _{t \rightarrow \infty}\left\{f^{1 / \gamma}\left(\frac{1}{R_{1}\left(t, t_{1}\right)}\right) \int_{t_{1}}^{t}\left(\frac{f\left(R_{1}\left(s, t_{1}\right)\right)}{r_{1}(s)} \int_{s}^{\infty} \tilde{Q}(u) \mathrm{d} u\right)^{1 / \gamma} \mathrm{d} s\right\}>k,
$$

then (1.1) has Property B.

Proof The proof is similar to that of Theorem 3 and so is omitted.

Remark 4 Note that in view of Lemma 6, the functions $Q(t)$ and $\tilde{Q}(t)$ can be replaced by

$$
Q_{n}(t)=\frac{p(t)}{r_{1}(t)} P_{n}(t)+q(t), \quad \tilde{Q}_{n}(t)=\frac{1}{r_{2}(t)} \int_{t}^{\infty} Q_{n}(s) \mathrm{d} s,
$$

respectively, for any $n \in \mathbb{N}$. 


\subsection{Oscillation of (1.1)}

If $g(t)>t$, we are also able to eliminate the remaining class of nonoscillatory solutions and ensure (1.1) to be oscillatory.

Theorem 5 Assume that all assumptions of Theorem 2 are satisfied and (2.13) holds. If

$$
\limsup _{t \rightarrow \infty} f^{1 / \gamma}\left(\frac{1}{R_{12}\left(g(t), t_{1}\right)}\right) \int_{t}^{g(t)} \frac{1}{r_{1}^{1 / \gamma}(x)}\left[\int_{t}^{x} \frac{1}{r_{2}(u)} \int_{t}^{u} R(s, t) \mathrm{d} s \mathrm{~d} u\right]^{1 / \gamma} \mathrm{d} x>k,
$$

then (1.1) is oscillatory.

Proof Let $y(t)$ be a positive solution of (1.1) on $\mathcal{I}$. It follows from Lemma 4 that there exists $t_{1} \in \mathcal{I}$ such that either $y(t) \in \mathcal{N}_{1}$ or $y(t) \in \mathcal{N}_{3}$ on $\left[t_{1}, \infty\right)$. From Theorem 2, we know that (1.1) has Property B, that is, $y(t) \in \mathcal{N}_{3}$. Integrating (1.1) from $t$ to $v$, we obtain

$$
L_{2} y(v) \geq L_{2} y(v)-L_{2} y(t) \geq \int_{t}^{v} \frac{p(s)}{r_{1}(s)} L_{1} y(s) \mathrm{d} s+\int_{t}^{v} q(s) f(y(g(s))) \mathrm{d} s .
$$

By Lemma 7, there exists $t_{2}>t_{1}$ such that $y(t) / R_{12}\left(t, t_{1}\right)$ is nondecreasing for $t \geq t_{2}$, and hence

$$
\begin{aligned}
L_{2} y(v) & \geq \int_{t}^{v} q(s) f(y(g(s))) \mathrm{d} s \\
& \geq f\left(\frac{y(g(t))}{R_{12}\left(g(t), t_{1}\right)}\right) \int_{t}^{v} q(s) f\left(R_{12}\left(g(s), t_{1}\right)\right) \mathrm{d} s, \quad t \geq t_{2} .
\end{aligned}
$$

Integrating (3.13) in $v$, one gets

$$
L_{1} y(v) \geq f\left(\frac{y(g(t))}{R_{12}\left(g(t), t_{1}\right)}\right) \int_{t}^{v} \frac{1}{r_{2}(u)} \int_{t}^{u} q(s) f\left(R_{12}\left(g(s), t_{1}\right)\right) \mathrm{d} s \mathrm{~d} u .
$$

Setting (3.14) into (3.12), we have

$$
\begin{aligned}
L_{2} y(v) & \geq f\left(\frac{y(g(t))}{R_{12}\left(g(t), t_{1}\right)}\right) \int_{t}^{v} \frac{p(s)}{r_{1}(s)} \tilde{q}(s, t) \mathrm{d} s+\int_{t}^{v} q(s) f(y(g(s))) \mathrm{d} s \\
& \geq f\left(\frac{y(g(t))}{R_{12}\left(g(t), t_{1}\right)}\right) \int_{t}^{v} R(s, t) \mathrm{d} s .
\end{aligned}
$$

Integrating in $v$ once more, we get

$$
L_{1} y(v) \geq f\left(\frac{y(g(t))}{R_{12}\left(g(t), t_{1}\right)}\right) \int_{t}^{v} \frac{1}{r_{2}(u)} \int_{t}^{u} R(s, t) \mathrm{d} s \mathrm{~d} u .
$$

Integrating in $v$ last time, we find

$$
y(v) \geq f^{1 / \gamma}\left(\frac{y(g(t))}{R_{12}\left(g(t), t_{1}\right)}\right) \int_{t}^{v} \frac{1}{r_{1}^{1 / \gamma}(x)}\left[\int_{t}^{x} \frac{1}{r_{2}(u)} \int_{t}^{u} R(s, t) \mathrm{d} s \mathrm{~d} u\right]^{1 / \gamma} \mathrm{d} x .
$$

Setting $v=g(t)$, we obtain

$$
\frac{y(g(t))}{f^{1 / \gamma}(y(g(t)))} \geq f^{1 / \gamma}\left(\frac{1}{R_{12}\left(g(t), t_{1}\right)}\right) \int_{t}^{g(t)} \frac{1}{r_{1}^{1 / \gamma}(x)}\left[\int_{t}^{x} \frac{1}{r_{2}(u)} \int_{t}^{u} R(s, t) \mathrm{d} s \mathrm{~d} u\right]^{1 / \gamma} \mathrm{d} x .
$$


Taking the lim sup on both sides of the resulting inequality, we are led to the contradiction with (3.11). Thus $\mathcal{N}_{3}=\emptyset$ and (1.1) is oscillatory. The proof is complete.

\section{Examples}

Example 1 Consider the third-order linear differential equation

$$
y^{\prime \prime \prime}(t)-\frac{a}{t^{2}} y^{\prime}(t)-\frac{b}{t^{3}} y(\lambda t)=0, \quad \lambda, a, b>0, t \geq 1 .
$$

A corresponding auxiliary equation

$$
v^{\prime \prime}(t)=\frac{a}{t^{2}} v(t)
$$

has a principal solution $v(t)=t^{\alpha}\left(\alpha=\frac{1}{2}(1-\sqrt{1+4 a})\right)$, which satisfies (2.6) if $a<2$. A simple calculation leads to

$$
\lim _{n \rightarrow \infty} Q_{n}(t)=\frac{b}{t^{3}}\left(1+\frac{a^{2}}{2(2-a)}\right) .
$$

Then, by Theorems 1 and 2 together with Remark 4, Property B of (4.1) is guaranteed if

$$
\begin{aligned}
& \frac{\lambda b}{2}\left(1+\frac{a^{2}}{2(2-a)}\right) \ln \left(\frac{1}{\lambda}\right)>\frac{1}{e} \quad \text { for } \lambda \in(0,1), \\
& \frac{b}{2}\left(1+\frac{a^{2}}{2(2-a)}\right) \ln \lambda>\frac{1}{e} \quad \text { for } \lambda>1 .
\end{aligned}
$$

By Theorems 3 and 4, the same conclusion holds for (4.1) if

$$
\begin{aligned}
& \frac{\lambda b}{2}\left(1+\frac{a^{2}}{2(2-a)}\right)>1 \quad \text { for } \lambda \in(0,1], \\
& \frac{b}{2}\left(1+\frac{a^{2}}{2(2-a)}\right)>1 \quad \text { for } \lambda \geq 1 .
\end{aligned}
$$

Furthermore, it follows from Theorem 5 that if $\lambda>1$ and

$$
\begin{aligned}
& b\left\{(1-2 a)\left(\frac{\lambda^{2}}{2} \ln \lambda-\frac{\lambda^{2}}{4}+\frac{1}{4}\right)-a(\lambda \ln \lambda-\lambda+1)\right. \\
& \left.\quad+(3 a-1)\left(\frac{\lambda^{2}}{2}-\lambda+\frac{1}{2}\right)+a\left(\frac{\lambda^{2}}{4} \ln ^{2} \lambda-\frac{\lambda^{2}}{4} \ln \lambda+\frac{\lambda^{2}}{8}-\frac{1}{8}\right)\right\}>1,
\end{aligned}
$$

then (4.1) is oscillatory.

Example 2 Consider the third-order nonlinear differential equation

$$
\left(\frac{1}{t} \sqrt[3]{y^{\prime}(t)}\right)^{\prime \prime}-\frac{a}{t^{3}} \sqrt[3]{y^{\prime}(t)}-\frac{b}{t^{10 / 3}} \sqrt[3]{y(\lambda t)}=0, \quad \lambda, a, b>0, t \geq 1 .
$$

Note that condition (2.5) is satisfied for $a \leq 4 / 3$. Then

$$
Q(t)=\left(\frac{9 a}{28}+b\right) \frac{1}{t^{10 / 3}},
$$


and by Theorems 1 and 2 we obtain that (4.2) has Property B provided that

$$
\begin{aligned}
& \frac{3}{7 \sqrt[3]{4}}\left(\frac{9 a}{28}+b\right) \sqrt[3]{\lambda^{4}} \ln \left(\frac{1}{\lambda}\right)>\frac{1}{e} \text { for } \lambda \in(0,1), \\
& {\left[\left(\frac{9 a}{28}+b\right) \frac{9}{28}\right]^{3} \ln (\lambda)>\frac{1}{e} \quad \text { for } \lambda>1 .}
\end{aligned}
$$

\section{Summary}

Very recently, authors suggested in $[8,12]$ the investigation of asymptotic and oscillatory properties for (1.1). Thus, in a certain sense, the presented results may be viewed as a complement of earlier obtained ones. We stress that, contrary to $[5,6,11]$, these criteria do not depend on solutions of the auxiliary equation (2.1).

For a particular case of (1.1), namely

$$
\left(r_{2}(t)\left(r_{1}(t) y^{\prime}(t)\right)^{\prime}\right)^{\prime}-q(t) y(t)=0
$$

Kusano et al. [18] have shown that there always exists a positive solution $y(t) \in \mathcal{N}_{3}$. If, however, $g(t)>t$, then we were able to eliminate also this class of solutions. It is clearly the advanced argument that can generate the oscillations.

It remains an open problem for further research to obtain the solution structure and corresponding asymptotic criteria for the equation

$$
\left(r_{2}(t)\left(r_{1}(t)\left(y^{\prime}(t)\right)^{\gamma}\right)^{\prime}\right)^{\prime}+p(t)\left(y^{\prime}(t)\right)^{\gamma}-q(t) f(y(g(t)))=0 .
$$

\section{Competing interests}

The authors declare that they have no competing interests.

\section{Authors' contributions}

Both authors contributed equally to this work. They all read and approved the final version of the manuscript.

\section{Acknowledgements}

We are grateful to the editors and three anonymous referees for a very careful reading of the manuscript and for pointing out several inaccuracies. The work on this research has been supported by the internal grant project No. FEI-2015-22.

Received: 21 November 2016 Accepted: 27 February 2017 Published online: 02 March 2017

\section{References}

1. Agarwal, RP, Grace, SR, O'Regan, D: Oscillation Theory for Difference and Functional Differential Equations. Springer, Media (2013)

2. Agarwal, RP, Grace, SR, O'Regan, D: Oscillation Theory for Second-Order Linear, Half-Linear, Superlinear and Sublinear Dynamic Equations. Springer, Media (2002)

3. Baculíková, B: Properties of third-order nonlinear functional differential equations with mixed arguments. Abstract and Applied Analysis 2011, Article ID 857860 (2011). doi:10.1155/2011/857860

4. Baculíková, B, Džurina, J: Properties of the third order trinomial functional differential equations. Electronic Journal of Differential Equations 2016, 244 (2016)

5. Baculíková, B, Džurina, J, Jadlovská, I: Properties of the third order trinomial functional differential equations. Electronic Journal of Qualitative Theory of Differential Equations 2015(34), 1-13 (2015)

6. Baculíková, B, Džurina, J, Rogovchenko, YV: Oscillation of third order trinomial delay differential equations. Applied Mathematics and Computation 218(13), 7023-7033 (2012)

7. Bartušek, M, Cecchi, M, Došlá, Z, Marini, M: Positive solutions of third order damped nonlinear differential equations. Mathematica Bohemica 136(2), 205-213 (2011)

8. Bohner, M, Grace, SR, Sager, I, Tunc, E: Oscillation of third-order nonlinear damped delay differential equations. Applied Mathematics and Computation 278, 21-32 (2016)

9. Bohner, M, Grace, SR, Jadlovska, I: Oscillation criteria for third-order functional differential equations with damping. Electronic Journal of Differential Equations 2016(215), 1 (2016)

10. Cecchi, M, Došlá, Z, Marini, M: On third order differential equations with property A and B. Journal of Mathematical Analysis and Applications 231, 509-525 (1999)

11. Džurina, J, Kotorová, R: Properties of the third order trinomial differential equations with delay argument. Nonlinear Analysis: Theory, Methods \& Applications 71(5), 1995-2002 (2009) 
12. Grace, SR: Oscillation criteria for third order nonlinear delay differential equations with damping. Opuscula Mathematica 35(4), 485-497 (2015)

13. Graef, J, Saker, SH: Oscillation theory of third-order nonlinear functional differential equations. Hiroshima Mathematical Journal 43, 49-72 (2013)

14. Gyori, I, Ladas, GE: Oscillation Theory of Delay Differential Equations: With Applications. Oxford University Press, London (1991)

15. Hartman, P: Ordinary Differential Equations. Willey, New York (1964)

16. Kiguradze, IT, Chaturia, TA: Asymptotic Properties of Solutions of Nonautonomous Ordinary Differential Equations. Kluwer Acad. Publ., Dordrecht (1993)

17. Kusano, T, Naito, M: Comparison theorems for functional differential equations with deviating arguments. J. Math. Soc. Jpn. 33(3), 509-532 (1981)

18. Kusano, T, Naito, M, Tanaka, K: Oscillatory and asymptotic behavior of solutions of a class of linear ordinary differential equations. Proc. R. Soc. Edinb. 90A, 25-40 (1981)

19. Koplatadze, R, Kvinkadze, G, Stavroulakis, IP: Properties A and B of n-th order linear differential equations with deviating argument. Georgian Math. J. 6, 553-566 (1999)

20. Ladde, GS, Lakshmikantham, V, Zhang, B: Oscillation Theory of Differential Equations with Deviating Arguments, vol. 110. Dekker, New York (1987)

21. Padhi, S, Pati, S: Theory of Third-Order Differential Equations. Springer, Berlin (2014)

22. Philos, ChG: On the existence of nonoscillatory solutions tending to zero at $\infty$ for differential equations with positive delays. Arch. Math. 36(2), 168-178 (1981)

23. Tiryaki, A, Aktaş, MF: Oscillation criteria of a certain class of third order nonlinear delay differential equations with damping. J. Math. Anal. Appl. 325, 54-68 (2007)

24. Trench, WF: Canonical forms and principal systems for general disconjugate equations. Trans. Am. Math. Soc. 189 319-327 (1974)

\section{Submit your manuscript to a SpringerOpen ${ }^{\circ}$ journal and benefit from:}

- Convenient online submission

- Rigorous peer review

- Immediate publication on acceptance

- Open access: articles freely available online

- High visibility within the field

- Retaining the copyright to your article 